\title{
Comprehensive metabolic engineering for fermenting glycerol efficiently in
}

$$
\text { Saccharomyces cerevisiae }
$$

\author{
Sadat M. R. Khattab ${ }^{1 *}, 2 *$, Takashi Watanabe ${ }^{1 *}$ \\ ${ }^{1}$ Research Institute for Sustainable Humanosphere, Kyoto University, 611-0011, Japan \\ ${ }^{2}$ Faculty of Science, Al-Azhar University, Assiut 71524, Egypt \\ *Correspondence to: khattab.sadatmohamedrezk.7c@kyoto-u.ac.jp, twatanab@rish.kyoto-u.ac.jp \\ 'Current addresses: ${ }^{1}$ Research Institute for Sustainable Humanosphere, Kyoto University, 611- \\ 0011, Japan
}

\section{ABSTRACT}

Glycerol is an eco-friendly solvent enhancing plant-biomass decomposition through a glycerolysis process in many pretreatment methods. Nonetheless, the lack of efficient conversion of glycerol by natural Saccharomyces cerevisiae restrains many of these scenarios. Here we outline the complete strategy for the generation of efficient glycerol fermenting yeast by rewriting the oxidation of cytosolic nicotinamide adenine dinucleotide (NADH) by $\mathrm{O}_{2}$-dependent dynamic shuttle while abolishing both glycerol phosphorylation and biosynthesis pathways. By following a vigorous glycerol oxidative pathway, the engineered strain demonstrated augmentation in conversion efficiency (CE) reach up to $0.49 \mathrm{~g}$-ethanol/g-glycerol— $98 \%$ of theoretical conversion-with production rate $>1 \mathrm{~g} / \mathrm{L}^{-1} \mathrm{~h}^{-1}$ when supplementing glycerol as a single fed-batch on a rich-medium. Furthermore, the engineered strain showed a new capability toward ferment a mixture of glycerol and glucose with producing $>86 \mathrm{~g} / \mathrm{L}$ of bioethanol with $92.8 \%$ of the CE. To our knowledge, this is the highest ever reported titer in this regard. Notably, this strategy flipped our ancestral yeast from non-growth on glycerol, on the minimal medium, to 
a fermenting strain with productivities $0.25-0.5 \mathrm{~g} / \mathrm{L}^{-1} \mathrm{~h}^{-1}$ and $84-78 \%$ of CE, respectively and $90 \%$ of total conversions to the products. The findings in metabolic engineering here may release the limitations of utilizing glycerol in several eco-friendly biorefinery approaches.

Keywords: Saccharomyces cerevisiae, bioethanol, glycerol fermentation, metabolic engineering, rewriting cofactors

\section{IMPORTANCE}

With the avenues for achieving efficient lignocellulosic biorefinery scenarios, glycerol gained keen attention as an eco-friendly biomass-derived solvent for enhancing the dissociation of lignin and cell wall polysaccharides during pretreatment process. Co-fermentation of glycerol with the released sugars from biomass after the glycerolysis expands the resource for ethanol production and release from the burden of component separation. Titer productivities are one of the main obstacles for industrial applications of this process. Therefore, the generation of highly efficient glycerol fermenting yeast significantly promotes the applicability of the integrated biorefineries scenario. Besides, the glycerol is an important carbon resource for producing chemicals. Hence, the metabolic flux control of yeast from glycerol contributes to generation of cell factory producing chemicals from glycerol, promoting the association between biodiesel and bioethanol industries. Thus, this study will shed light on solving the problems of global warming and agricultural wastes, leading to establishment of the sustainable society.

\section{INTRODUCTION}

One of the challenges for sustaining the future humanosphere is to produce required biobased chemicals and fuels from renewable resources to reduce greenhouse gas emissions. A 
massive requirement for ethanol recently arose for use in sanitizers because of the COVID-19 pandemic. Bakers' yeast (Saccharomyces cerevisiae) has several desirable characteristics during bioethanol production, such as the high producer with a long and safe history of use. Besides, its unicellular structure, short life cycle, remarkable tolerance to inhibitors and stress during the industrial processes. Hence has been selected as a top model platform of microbial cell factories for several biotechnological applications $(1,2)$.

The resources of fermentable sugars are limited. Therefore, challenges exist to overcome the drawbacks of production from lignocellulosic biomass through evolving the maximum efficiencies in ethanol production from xylose and acetic acid with glucose (3-5). In the past decade, glycerol-producing industries, particularly that of biodiesel, have expanded and accumulated substantial amounts of glycerol, which has led to price drops (6). Although the reduction degree of glycerol $\left(\mathrm{C}_{3} \mathrm{H}_{8} \mathrm{O}_{3}\right)$ is higher than that of other fermentable sugars (7), glycerol is classifying as a non-fermentable carbon in native $S$. cerevisiae (2). Additionally, glycerol is a carbon source poorly utilized primarily via the glycerol-3-phosphate pathway (herein referred to as the G3P pathway), which is composed of glycerol kinase (GUT1) and FAD-dependent-mitochondrial glycerol-3-phosphate dehydrogenase (GUT2) (8). Yeast biosynthesis glycerol for mitigating osmotic stress and optimizing the redox balance (9). Furthermore, glycerol catabolism is subject to the repression and transcriptional regulation of glucose through respiratory factors and the GUT1 and GUT2 genes (10-12).

The trials to ferment glycerol in S. cerevisiae by overexpressing the native oxidative pathway (DHA; dihydroxyacetone pathway) have been reported. Overexpress endogenous glycerol dehydrogenase $(S c G C Y 1)$ with dihydroxyacetone kinase $(S c D A K 1)$ produced $0.12 \mathrm{~g}$ ethanol/ $\mathrm{g}$ glycerol $\left(\mathrm{g}^{\mathrm{e}} / \mathrm{g}^{\mathrm{g}}\right)$. The production rate was $0.025 \mathrm{~g} / \mathrm{L}^{-1} \mathrm{~h}^{-1}(13)$. The importance of glycerol as a 
carbon source that can be utilized by yeast cells has further prompted the study, which revealed the intraspecies diversity ranging from good glycerol grower to non-growers among $52 S$. cerevisiae studied strains. The growth phenotype is controlled by quantitative traits (14). It has been reported that many alleles, such as cytoplasmic ubiquitin-protein ligase E3, UBR2, link GUT1 for growth on glycerol in synthetic medium without supporting supplements (15). On the other hand, heterologous replacement of G3P with DHA combined with the glycerol facilitator (FPS1) resulted in the restoration of growth characteristics like the parental strain or even higher (16). This replacement in a positive-glycerol grower ancestor with limiting the oxygen in shaking flask cultures increased the ethanol production 0.165 to $0.344 \mathrm{~g}^{\mathrm{e}} / \mathrm{g}^{\mathrm{g}}$ in buffered synthetic medium. The maximum titer produced reached $15.7 \mathrm{~g} / \mathrm{L}$ after $144 \mathrm{~h}(17)$. So far, glycerol is reporting as a non-fermentable carbon source in S. cerevisiae, and attempts are undergoing to ferment this carbon in this yeast (2). Meanwhile, the methylotrophic yeast Ogataea polymorpha has been tested for bioethanol production from glycerol by overexpressing multiple genes involved in either the DHA or G3P pathways. In addition to FPS1, pyruvate decarboxylase $(P D C) 1$, and alcohol dehydrogenase $(A D H)$ 1. The overall ethanol production was $10.7 \mathrm{~g} / \mathrm{L}$ ethanol as a maximum accumulated product at an efficiency of $0.132 \mathrm{~g}^{\mathrm{e}} / \mathrm{g}^{\mathrm{g}}$. (18). Hitherto, there is no known native or genetically engineered $S$. cerevisiae strain ferments glycerol efficiently to ethanol.

Of note, we previously reported microwave-assisted pretreatments of recalcitrant lignocellulosic biomass in aqueous glycerol (19) and further innovated that method with the catalysis of alum [AlK(SO4)2] (20). Fractionation of sugars from glycerol is a costly process. Therefore, to improve our scenario, there is a need for developing an S. cerevisiae capable of efficiently fermenting glycerol with glucose following glycerolysis of biomass. Such glycerolfermenting strain will co-ferment glycerol to synergist ethanol production from hydrolyzed 

lignocellulosic biomass to an economical distillation process. In the present study, we report the details of modelling the yeast cell to redirect glycerol traffic to bioethanol production to the highest ever reported $>8.6 \%$ - with the presence of glucose-through innovation of the forthcoming systematic metabolic engineering outlined in Figure 1 as follows: 1) abolishment of the inherent glycerol biosynthesis pathway by knocking out NAD-dependent glycerol-3phosphate dehydrogenase $(G P D) 1 ; 2$ ) replacement of cytosolic NADH oxidation through the ScGPD1 shuttle with a more effective $\mathrm{O}_{2}$-dependent dynamic shuttle of water forming the NADH oxidase NoxE; 3) knocking out the first gene of the G3P pathway (GUT1); and 4) implementing a vigorous oxidative pathway via overexpression of two copies of both the heterologous genes of glycerol dehydrogenase from $O$. polymorpha $(\mathrm{Op} G D H)$ and the glycerol facilitator CUFPS1, besides two copies of both the endogenous genes ScTPI1 and ScDAK1 with one copy of $S c D A K 2$.

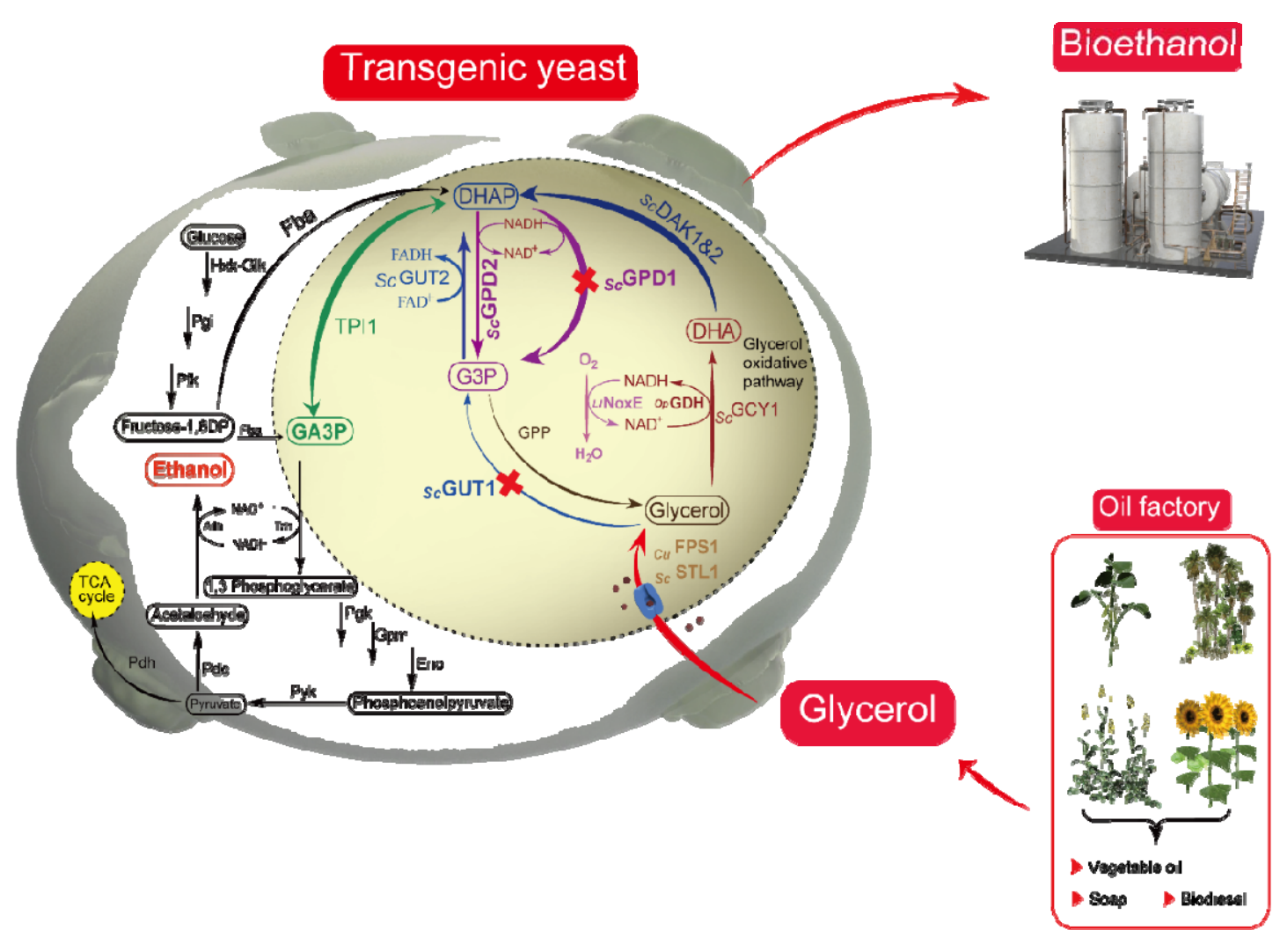




\section{MATERIAL AND METHODS}

Strains, primers, cassettes, and plasmids constructions. Ancestor and all recombinant strains used in this study are listed in Table 1. The original ancestors showed in Fig. S1. The plasmids used in this study are listed in Table 2. The primers used here are listed in Table S1, and those for obtaining the native genes were designed based on the sequences available on the S288C Saccharomyces Genome Database (SGD): https://www.yeastgenome.org/ (S288C). Details of DNA fragments, cassettes, and plasmids construction are explained as follows:

Construct pPGK-ScTPI1, ScDAK2, ScDAK1 plasmids. We obtained the genes from genomic DNA of the ancestor strain D452-2 to clone the plasmids in this section. Initially, cell walls were disrupted by resuspension with picked cells in $20 \mu 1$ of $30 \mathrm{mM} \mathrm{NaOH}$ at $95^{\circ} \mathrm{C}$ for 10 min, and then used directly as a template for PCR; $1 \mu 1$ of freshly disrupted cells are suitable for a $50 \mu 1$ of PCR mixture. High fidelity polymerisation of KOD-plus neo with their corresponded primers (Table S1, section 1) was used during this amplification. The Xhol site of DAK2 was deleted before cloning. Genes were purified from the PCR mixtures FastGene Gel/PCR Extraction Kit (Nippon Genetics Co. Ltd), and their cohesive ends were formed according to the designated primers and restriction enzymes. We first separately cloned each gene in the pPGK/URA3 plasmid (21) under the control of the expression system PGK promoter and PGK terminator (Table 2). We obtained the plasmids and confirmed their gene sequences via sequencing using primers (Table S1, section 2). Next, we cut the XhoI/SalI-ScDAK2 cassette and inserted it into the SalI site of the pPGK-ScTPI1 plasmid. The non-reopened ligations (Xhol/SalI sites) were used during the ligation of new cassettes of DAK1 to form the new plasmid (Table 2). 

section 3). Then, cohesive ends of those coupled DNA fragments were processed by the restriction enzymes XhoI, NotI for the first fragment, and NotI, SalI for the second. After purification of the fragments, one-step cloning was employed coupled the TDH3 promoter and mutated DITI terminator into XhoI/SalI of PGK/URA3 plasmid. Then the TDH3p-d22DIT1tURA3 plasmid was constructed (Table 2). Subsequently, we cloned the $O p G D H$ gene into the constructed TDH3-d22-DIT1t plasmid to form the TDH3-OpGDH-d22DIT1t- plasmid. Synthetic the accession number XP_018210953.1. Full sequences are available in Table S2. Also, we bought the water-forming NADH oxidase gene of Lactococcus lactis subsp. lactis Il1403 (IDT) based on the sequence on gene bank accession number AAK04489.1. Then cloned it into TDH3p-d22DIT1t to assemble TDH3-d22- LINoxE plasmid (Tables 2).

145 (23). We used an online tool for the rational design of CRISPR/Cas target to allocate the highest 146 probability of the on-target sites for the gRNA in the genomic DNA of $S$. 147 cerevisiae (https://crispr.dbcls.jp/) (24). Accordingly, the primers were designed based on the 
148

149

150

151

152

153

154

155

156

157

158

159

160

161

162

163

164

165

166

167

168

169

170

previously allocated sequence (20 bp before the PAM), with another $20 \mathrm{bp}$ from sgRNA or HDV ribozyme for overlap (Table S2, sections 3.2, and 6.1). First, PCR was used to synthesize two fragments from the template, the pCAS-gRNA plasmid. The first fragment was amplified using the forward primer called pCAS F-located upstream of the gRNA scaffold at the SmaI site of the multiplex plasmid - and antisense primer with a reverse sequence of the target gRNA. The second fragment was amplified by a forward primer, which has a sense sequence of gRNA, and the reverse primer called pCAS R-located downstream of the gRNA scaffold (Table S1, section 3.2 and 6.1). After purifying each DNA segment, overlapping and integration were carried out by PCR using the pCAS F and R primers. The produced fragment was then end-repaired to the SmaI-PstI sites for cloning into a truncated pCAS-gRNA plasmid with SmaI-PstI. As a result, a new multiplex pCAS-gRNA plasmid was formed. Steps have been repeatedly performed with constructing multiplex pCAS-gRNA plasmids targeting $S c G P D 1, G U T 1$ genes (Table 2). We confirmed the newly constructed systems by sequencing their entire scaffolds.

\section{Construct pAUR101- CuFPS1 and pAUR101-CuFPS1, ScTPI1, ScDAK2, ScDAK1}

plasmids. Candida utilis (NBRC 0988) was obtained from the National Biological Resource Center (NBRC) of the National Institute of Technology and Evaluation (Japan) and was used as a template to get the gene glycerol facilitator FPS1 (CuFPS1). The sequence of CuFPS1 was included in the deposited gene bank accession number BAEL01000108.1. The original pAUR101 plasmid was purchased from Takara Bio, Inc., Japan, and the primers were used to establish this plasmid listed (Table S1, section 4). A full sequence for the cassette PGK-CuFPS1RPL41Bt was showed (Table S2). First, we constructed a pAUR101-PGKp-RPL41Bt vector by one-step cloning of the SmaI-Not1 PGK promoter (fragment F1) and NotI-SacI-RPL41B terminator (F2) into the SmaI-SacI pAUR101 vector and then cloning a cohesive ended NotI- 
CuFPS gene into the dephosphorylated NotI site of pAUR101-PGK-RPL41B vector to assemble pAUR101-PGKp-CuFPS1-RPL41Bt vector. We confirmed the clone direction by PCR. To constitute the pAUR101-CuFPS1, ScTPI1, ScDAK2, ScDAK1 plasmid, we detached the set of cassettes-ScTPI1, ScDAK2, and ScDAK1—from the previously constructed plasmid, pPGKScTPI1, ScDAK2, and ScDAK1 (Table 2) using restriction enzymes Xhol-SalI and re-inserted that set of cassettes into the SalI site of the pAUR101-PGK-CuFPS1-RPL41B plasmid (Table 2).

Construct Module M1 and pAUR101- M1 plasmid. We first obtained all fragments which could form the module M1 separately by PCR (Fig. 2); the CuFPS1, OpGDH genes, and mutated d22DIT terminator amplified from their synthetic DNA stocks, whereas other fragments were magnified from the genomic DNA of the D452-2 strain (25). The full sequence of the module M1 is also accessible (Table S2), and the primer details are listed in Table S1, Section 5. Purification of the 12 amplified DNA pieces was carried out on 1\%-2\% agarose gel and then recovered by the extraction Kit. We accordingly obtained highly purified fragments before the onset of assembly using the Gibson Assembly Master Mix. We first joined every three consecutive segments seamlessly according to the manufacturer's protocol (Gibson). We also directly amplified each set by PCR and then purified these again on an agarose gel. We repeatedly gathered every six sequential segments and then assembled the whole module M1; TEFp-CuFPS1-CYC1t; TYS1p-OpGDH-ATP15t; TDH3p-ScDAK1-d22-DIT1t; FBA1pScTPI1-TDH3t (Fig.2). We further added the SacI site upstream of the module M1 and the SmaI site downstream. These restriction sites were provided for cloning the module M1 into SacISmaI sites of pAUR101 vector to form pAUR 101-M1 (Table 2). Finally, we transferred the vector pAUR 101-M1 into E. coli as described previously and confirmed the accurate structure of M1 by sequencing the whole module M1 from pAUR-M1. 
194

195

196

197

198

199

200

201

202

203

204

205

206

207 208
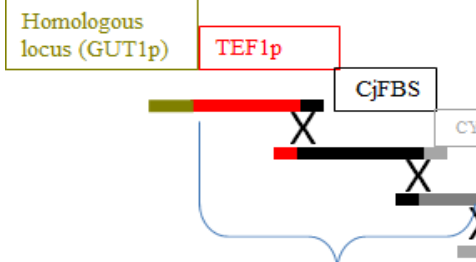

Candida utilis glycerol facilitator cassette

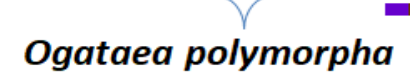
glycerol dehydrogenase cassette

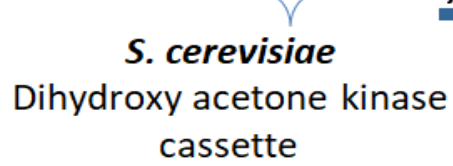

cassette

\section{S. cerevisiae}

Triose phosphate isomerase

cassette

Transformation and recombination of strains. All the previous plasmids were stored in $E$. coli NEB 10-beta for further use, where the heat shock method was used during the transformation according to the procedures provided with the competent cells. All plasmid extractions were performed using the QIAprep Spin Miniprep Kit following the manufacturer's protocol. All measurement of DNA was estimated using BioSpec-nano (Shimadzu, Japan). DNA was stored at $-20^{\circ} \mathrm{C}$ for future use. Yeast transformation was carried out by Fast Yeast Transformation ${ }^{\mathrm{TM}}$ kit (Takara Bio) to integrate linear pAUR101 vector and its associated genes in the AUR1-C locus. Also, linear pPGK plasmid with its cloned genes in URA3 loci (25). For achieving genome editing and the replacement of $S c G P D 1$ and $S c G U T 1$ genes with its designated DNA repairing cassette or module, we used the protocol of CRISPR-Cas9 genome engineering in $S$. cerevisiae cells (26). We confirmed target replacements using PCR for the inserted repairing cassettes with primers from upstream and downstream of the flanking recombined loci. We re-confirmed the recombination after the losses of pCAS multiplex plasmid. All recombination strains and their genotypes are listed in Table 1. 
Preparation of cell-free extract. Cell proteins were extracted as previously described $(3,27)$ with some modifications. The recombinant strains listed in Table 1 were cultivated in closed 50$\mathrm{ml}$ Falcon tubes with $10 \mathrm{ml}$ of YP medium supplemented with (w/v) $1.5 \%$ glucose and $7 \%$

212 glycerol for $15 \mathrm{~h}$. Cell pellets were harvested by centrifugation at $700 \mathrm{xg}$ for $2 \mathrm{~min}$ at $4{ }^{\circ} \mathrm{C}$, then 213 washed with $20 \mathrm{ml}$ of $100 \mathrm{mM}$ of HEPES buffer (pH 7.4) and centrifuged again. Then, the cell 214 pellets were lysed in $1 \mathrm{ml}$ of HEPES buffer supplemented with $1 \mathrm{mM} \mathrm{MgCl}$ and $10 \mathrm{mM} 2-$ 215 mercaptoethanol with approximately $400 \mathrm{mg}$ of glass beads in a 2-ml Eppendorf tube. The lysis 216 was accomplished by vigorous shaking by bench vortex with six-time intervals on ice for 30 217 seconds. The crude proteins were separated from the glass beads and cell debris by two rounds of centrifugation at $22300 \mathrm{xg}$ at $4{ }^{\circ} \mathrm{C}$ for $5 \mathrm{~min}$. Total protein concentration was estimated using Bioas a standard. change (UV-2700; UV-VIS spectrophotometer, Shimadzu, Japan). One ml mixture of $80 \mathrm{mM}$ HEPES buffer (pH 7.4), $5 \mathrm{mM} \mathrm{NAD}{ }^{+}, 100 \mathrm{mM}$ glycerol, and $10 \mu \mathrm{l}$ of crude extract were mixed during analysis. The activity of ScTPII was assayed as described previously (28) with some modifications. The reaction mixture was $1 \mathrm{ml}$ in volume and was composed of $100 \mathrm{mM}$ 227 triethanolamine hydrochloride $(\mathrm{pH} 7.53), 2.5 \mathrm{mM} \mathrm{NAD}^{+}, 10 \mathrm{mM}$ DHAP, and $10 \mu \mathrm{l}$ of crude extract. Dihydroxyacetone kinase was assayed using a universal kinase activity kit according to the manufacturer's instructions (Catalog Number EA004). 

EnzyChrom $^{\mathrm{TM}} \mathrm{NAD}^{+} / \mathrm{NADH}$ Assay Kit.

Fermentation procedures. The fermentation experiments were performed in several conditions defined as following according to the ratio of liquid medium (ml): Erlenmeyer flasks volume (ml). Micro-aerobic [20:100], semi-aerobic [20:200], aerobic [20:300]. We conducted the fermentation at $30 \pm 2{ }^{\circ} \mathrm{C}$ with agitation speeds at 180 or $200 \mathrm{rpm}$. For strict anaerobic conditions, $20 \mathrm{ml}$ of YNB medium with initial cell pellets were transferred into a sterilized 50-ml vial and then capped with a precision seal septa cap. Afterward, the nitrogen gas was flushed into the culture medium through Terumo needles. Sampling was drawn through needles. The

240 fermenter cells were harvested from the same volume of the pre-culture YPD medium for 241 approximately $15 \mathrm{~h}$. For $\mathrm{SK}-\mathrm{FGG}$, the cultivation culture medium was $\mathrm{YPD}_{20} \mathrm{G}_{70}$. Cells were 242 harvested by centrifugation at $1600 \times \mathrm{g}$ for $5 \mathrm{~min}$ at $4^{\circ} \mathrm{C}$ and washed with sterile water. Then, 243 collected cells were re-supplemented with the YP medium with glucose, glycerol, or both, as 244 shown in figures and tables. These concentrations have been chosen to match those can obtain 245 after the glycerolysis of biomass (20). The cell density was monitored using spectrophotometry 246 at $600 \mathrm{~nm}$ (AS ONE, Japan).

247 Fermentation analysis. All analyses were performed using ultra-fast liquid chromatography 248 (Shimadzu, Japan). Automatic sampling was fractionated by Aminex HPX-87H column (Bio249 Rad Laboratories, Hercules, CA, USA), then analyzed in a refractive index detector (RID-10A; 250 Shimadzu) and a prominence diode array detector (SPD-M20A; Shimadzu). Fractionation was 251 accomplished at a flow rate of $0.6 \mathrm{ml} / \mathrm{min}$ with $5 \mathrm{mM} \mathrm{H}_{2} \mathrm{SO}_{4}$ as the mobile phase at $50^{\circ} \mathrm{C}$. 252 Reactant concentrations were estimated by monitoring the peak areas compared with the 
standards of the authenticating reactant's glucose, glycerol, ethanol, acetic acid, pyruvate, succinic acid, and acetaldehyde, which detected by RID. We used the SPD to estimate DHAP and DHA. The detectable quantities were reported in the tables or figures and were omitted if it was undetectable.

\section{RESULTS}

Overexpress of a vigorous glycerol dehydrogenase. The overexpression of $O p G D H$ in the GDH strain was verified by comparing the enzyme activity with the reference D452-2 (Table 3). As a result, $58 \%$ of glycerol was consumed compared with $8 \%$ from reference strain, which raised ethanol level from 4.47 in ancestral to $11.82 \mathrm{~g} / \mathrm{L}$ in $\mathrm{GDH}$ strain, representing $0.27 \mathrm{~g}^{\mathrm{e}} / \mathrm{g}^{\mathrm{s}}$ before reutilizing the produced ethanol after 26h (Fig. 3).

Integrate the replacement of $S c G P D 1$ by $L I N o x E$ with $O p G D H$. Based on data from the comparative study of substituting GPD shuttles with LINoxE, the replacement of ScGPD1 by LINoxE abolished glycerol biosynthesis by $98 \%$ (Khattab and Watanabe, manuscript in preparation). Therefore, we studied the recycling outputs of $\mathrm{NAD}^{+} / \mathrm{NADH}$ between $O p G D H$ and LINoxE genes. We projected abolishing glycerol formation and decreases the ramification of DHAP, which consolidates straightforward progression to the glycolysis route and ethanol formation. This innovative integration clearly showed improvements in glycerol conversion efficiency to ethanol and delay in cell shift to utilize the produced ethanol. The ethanol production boosted to $13.27 \mathrm{~g} / \mathrm{L}\left(0.31 \mathrm{~g}^{\mathrm{e}} / \mathrm{g}\right.$ substrate consumed $)$ and extending the fermentation time to $32 \mathrm{~h}$ with further raising the ethanol titer to $14.42 \mathrm{~g} / \mathrm{L}$. (Fig. 3). Analysis of a cofactor ratio ( $\mathrm{NADH} / \mathrm{NAD}^{+}$) showed a significant change from 0.68 to 0.99 between GDH and GN strains, respectively (Table.3). The higher NADH/NAD ${ }^{+}$ratio is pointing to the higher efficiency of replacing ScGPD1 with LINoxE shuttle. 

current outputs, further limitations in the activity of other genes in the DHA pathwayScTPI1, ScDAKs, and CUFPS1-affecting the traffic of glycerol conversion to ethanol. Therefore, we overexpressed all of them. In this point, we clarify that ScDAK2 and ScTPII are not considered during the previous studies of converting glycerol to ethanol. In the GN-FDT strain, the specific enzyme activity of $O p G D H$ increased by $9 \%$ compared with the GN strain. Also, ScDAKs and ScTPII increased by $83 \%$ and $16 \%$, respectively, compared with the ancestor strain. Moreover, the $\mathrm{NADH} / \mathrm{NAD}^{+}$ratio decreased to $0.33 \%$ of the GN (Table 3 ). This recombination step (GN-FDT strain) unequivocally solved one of the problems in this study, where it prevented the shift in utilizing ethanol before the consumption of glycerol. The consumption rate reached $1 \mathrm{~g} / \mathrm{L}^{-1} \mathrm{~h}^{-1}$ and produced $20.95 \mathrm{~g} / \mathrm{L}$ of ethanol using this recombinant value (Fig. 3).
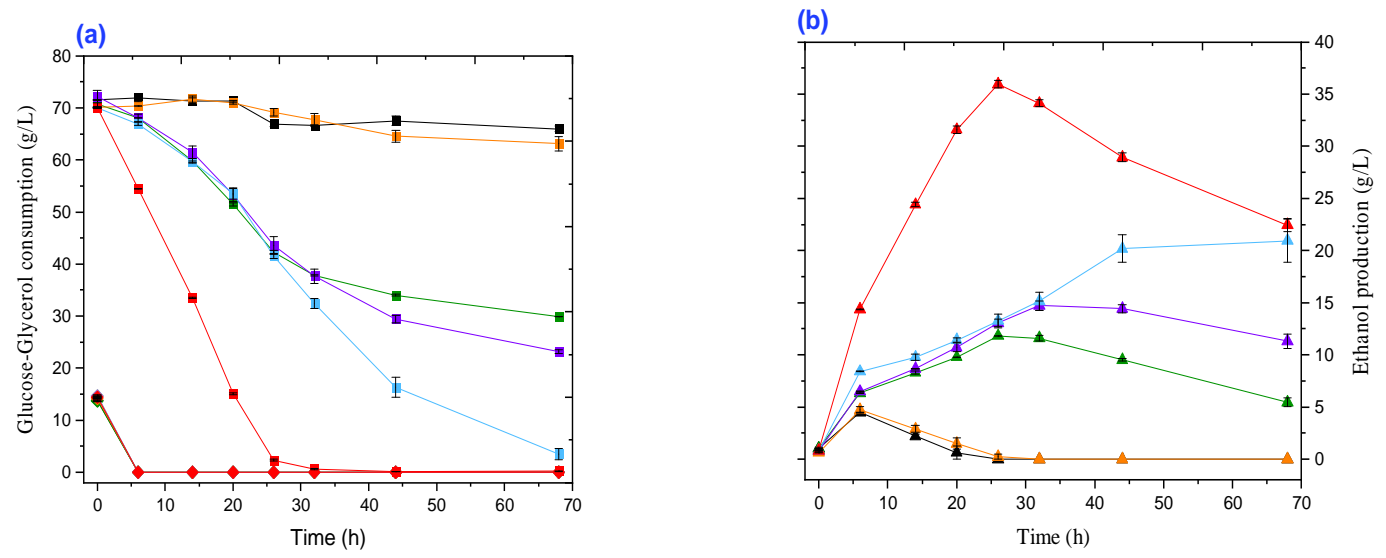
constructed by employing Gibson hybrid assembly and PCR to replace ScGUT1 (Fig. 2). As a result of integration with replacement, the specific enzyme activities of $O p G D H, S c D A K$, and ScTPII were further enhanced by $56 \%, 256 \%$, and $13 \%$, respectively, compared with the GNFDT strain. Besides, the $\mathrm{NADH} / \mathrm{NAD}^{+}$ratio decreased to 0.11 (Table 3). Interestingly, we obtained outstanding fermentation in this fourth step of recombination GN-FDT-M1, named SKFGG strain (Table 1, Fig. 3). The glycerol consumption rate reached $2.6 \mathrm{~g} / \mathrm{L}^{-1} \mathrm{~h}^{-1}$ at the described experimental conditions, and the productivity paced $1.38 \mathrm{~g} / \mathrm{L}^{-1} \mathrm{~h}^{-1}$ of ethanol with a conversion efficiency of $0.44 \mathrm{~g}^{\mathrm{e}} / \mathrm{g}^{\mathrm{s}}$ (Fig. 3).

Ferment powers in the engineered yeast. With the current state of metabolic engineering, we examined fermentation characteristics at higher initial concentrations of glycerol $(110 \mathrm{~g} / \mathrm{L})$ in the absence and presence of glucose $(22.5 \mathrm{~g} / \mathrm{L})$, where glucose was reported as a suppressor for glycerol fermentation (Table 4 [A and B]). We also tested fermenting a further fed-batching of $100 \mathrm{~g} / \mathrm{L}$ glycerol to the condition of $[\mathrm{B}]$ in (Table $4[\mathrm{C}]$ ). To further confirming the engineered strain is unsubjected to repression by glucose during the glycerol fermentation, we lifted the glucose level to $45 \mathrm{~g} / \mathrm{L}$ with decreased the glycerol concentration by $25 \%$ to $82 \mathrm{~g} / \mathrm{L}$ before further adding $100 \mathrm{~g} / \mathrm{L}$ glycerol as a fed-batch (Table 4 [D]). These outlines also testing the strain SK-FGG to produce an economically distillable ethanol titer (Table 4 [D]). The concentrations of glucose and glycerol in [D] are relatively like those obtained after the glycerol process (20). The strain SK-FGG exhibited outstanding performance in semi-aerobic conditions at a higher initial concentration of glycerol-glucose mixture. Its conversion efficiency reached $98 \%\left(0.49 \mathrm{~g}^{\mathrm{e}} / \mathrm{g}^{\mathrm{g}}\right)$ with a production rate of $>1 \mathrm{~g} / \mathrm{L}^{-1} \mathrm{~h}^{-1}$ of ethanol after consumed $82.5 \mathrm{~g} / \mathrm{L}$ of glycerol from one fed-batch condition (Table 4, condition [A]). Acetic acid accumulated at 1.14 $\mathrm{g} / \mathrm{L}$ at this condition (Table 4, condition [A]). Even at mixing glycerol with $22.55 \mathrm{~g} / \mathrm{L}$ of glucose, 
its conversion efficiency was comparatively the same (Table 4, condition [B]). Interestingly, the strain engineered here is exceptional in its capacity to harmonize fermenting glycerol with glucose, along with an accumulation of $>86 \mathrm{~g} / \mathrm{L}$ of bioethanol with additional fed batching of glycerol (Table 4, [D]). With higher initial glucose at condition [D], cell density was promoted by $31 \%$ compared with the case $[\mathrm{C}]$; besides, a minor reduction of the efficiency of ethanol conversion (Table 4, [C and D]).

Ferment glycerol as a sole carbon source. Eventually, verifying the fermentation capability of SK-FGG has been conducted on glycerol as a sole carbon in a yeast nitrogen base (YNB) medium with only $20 \mathrm{mg} / \mathrm{L}$ of leucine and histidine in a single-batch. We compared the fermentation at four degrees of oxygen availabilities. Under the strict anaerobic conditions, there was no further growth nor production of ethanol (Table 5). Under micro-aerobic conditions, the strain consumed $37.17 \mathrm{~g}$ glycerol with a consumption rate that reached $0.62 \mathrm{~g} / \mathrm{L}^{-1} \mathrm{~h}^{-1}$ and a production rate of $0.25 \mathrm{~g} / \mathrm{L}^{-1} \mathrm{~h}^{-1}$. The efficiency of ethanol conversion approached $0.42 \mathrm{~g}^{\mathrm{e}} / \mathrm{g}^{\mathrm{g}}$. Acetic acid accumulated at $0.78 \mathrm{~g} / \mathrm{L}$ in this condition (Table 5). Glycerol was consumed more rapidly in a semi-aerobic atmosphere. The consumption rate was $>1 \mathrm{~g} / \mathrm{L}^{-1} \mathrm{~h}^{-1}$, which raised the ethanol production rate to $0.44 \mathrm{~g} / \mathrm{L}^{-1} \mathrm{~h}^{-1}$ till accumulate $20.97 \mathrm{~g} / \mathrm{L}$. There was $2.88 \mathrm{~g}$ of acetic acid cumulation in this condition. As a result, the total conversion reached $0.44 \mathrm{~g} / \mathrm{g}$ glycerol (Table 5). In aerobic conditions, glycerol consumption and ethanol production were boosted to 1.29 and $0.5 \mathrm{~g} / \mathrm{L}^{-1} \mathrm{~h}^{-1}$, respectively. The efficiency of ethanol conversion was $0.39 \mathrm{~g}^{\mathrm{e}} / \mathrm{g}^{\mathrm{g}}$, and the total convertibility was $0.45 \mathrm{~g}^{\mathrm{e}} / \mathrm{g}^{\mathrm{g}}$, which represents $90 \%$ of the theoretical conversion regardless of the utilized glycerol in cell formation (Table 5).

\section{DISCUSSION}


The ancestral capability to grow on glycerol. The ancestor strain showed no growth (a maximum $\mathrm{OD}_{600}$ after $180 \mathrm{~h}$ was 0.28 ) on glycerol as a sole carbon provided in YNB medium (without amino acids) supplemented by $20 \mathrm{mg} / \mathrm{L}$ of leucine, histidine, and $5 \mathrm{mg} / \mathrm{L}$ of uracil. One of the parent ancestors $(25,29,30)$ belongs to S288C. Swinnen et al. reported that the S288C strain is classifying as a negative glycerol grower on a synthetic medium without supporting supplements (14). Hence, herein we initially focused on using a conventional YP medium to avoid these limitations.

Comprehend the modeling of glycerol fluxes to bioethanol in S. cerevisiae. Introducing an active $O p G D H$ gene is the first key for deciphering glycerol fermentation, where the activity of endogenous glycerol dehydrogenases was not observed (Table 3). Overexpressing ScGCY1 alone or combined with the whole DHA is much lower than $O p G D H$ here (Data not shown). Although the sole overexpresses of $O p G D H$ was not enough to induce efficient conversions (Fig. 3), where the raises of $\mathrm{NADH} / \mathrm{NAD}^{+}$ratio by $45 \%$ (Table 3 ) presumably promote oxidizing this plethora through the other NADH-dependent shuttles. At this juncture, Heterologous overexpressing a more dynamic shuttle with the reduced circulation of DHAP into glycerol biosynthesis or/and

353 G3P — glycerolipid — was confirmed as a new point for support fermentation of glycerol or/with glucose. Heterologous expression NoxE in S. cerevisiae has been successfully oxidizing the excess of NADH during glucose fermentation to produce ethanol or 2,3-butanediol or acetoin. substantial improvement in ethanol production, which reached $28 \%$ compared with the GDH 358 strain within the studied conditions (Fig. 3). This effectiveness of replacing ScGPD1 with LINoxE shuttle was further confirmed by restoring the balance of intracellular NADH/NAD ${ }^{+}$ 360 ratio (Table 3 ). 
Also, DHAP has been reported for distribution partially into phospholipid and methylglyoxal biosynthesis $(36,37)$. Therefore, further reducing the ramification of DHAP could be overcome with the overexpressing the ScTPI1. The pivotal role of ScTPII is also known toward glycerol production (38). Moreover, overexpressing ScDAK2 with ScDAK1 and CuFPS1(39) supported the fermentation of glycerol to ethanol. Overexpressing $S c D A K 2$ (which has a much lower $\left.K m_{\text {(DHA- ATP) }}\right)$ with $S c D A K 1$ was reported to detoxified the DHA (40). By integrating the genes in GN-FDT strain, continued bioethanol production until the consumption of glycerol is completed. Nonetheless, the ethanol titer was $20.95 \mathrm{~g} / \mathrm{L}$ represents less than $48 \%$ of fermentation efficiency (Fig.3). Owing to enhanced activities of ScTPII, ScDAKs, OpGDH, and enhance the channeling of glycerol, we overcame re-consuming the produced ethanol earlier than the occurrence of consumption of glycerol (Fig. 3). Thus, the increased activities of ScDAKs with ScTPII represent another essential step with both $O p G D H$ and CuFPS1 for glycerol conversion to ethanol. Moreover, the lowering of NADH/NAD ${ }^{+}$ratio (Table 3).

Overexpress multi-copies of DHA pathway. With scrutinizing the result, we recognized the conversion efficiency may still be affected by the robustness of natively programmed glycolysis toward glycerol biosynthesis. Furthermore, the activity of alcohol dehydrogenase (ADH) within cells grown in glycerol yeast extract medium has been shown to be ten times higher as compared to those cultured in glucose (41). For overcoming this, we investigated the reinforces the influxes of glycerol with another copy of the DHA pathway. Besides, support the availability of ATP for $S c D A K s$ through replacing a phosphorylation pathway through GUT1 (16). Here, with the integration of the second copy of genes CuFBS1, OpGDH, ScDAK1, and ScTPI1 with highly constitutive expressing systems during replacing $\operatorname{Sc} G U T 1$ (22, 42, 43), we extend production levels and efficiencies from $0.85-1.28 \mathrm{~g} / \mathrm{L}^{-1} \mathrm{~h}^{-1}$ and the conversion efficiency reached $98 \%$ of the 
theoretical ratio in the YP medium (Table $4[\mathrm{~A}]$ ). Furthermore, the strain overrode the glucose repression during glycerol fermentation with glucose and produced up to $8.6 \%$ of ethanol (Table $4[\mathrm{D}])$.

Ferment glycerol as a sole carbon. Interestingly, flipped her nature even from non-growing on glycerol with this systematic metabolic engineering, where SK-FGG strain showed abilities to convert glycerol in YNB medium—without supplementary amino acids. In micro and semiaerobic conditions, ethanol production rates were 0.25 and $0.44 \mathrm{~g} / \mathrm{L}^{-1} \mathrm{~h}^{-1}$ with efficiencies of 0.42 and $0.39 \mathrm{~g}^{\mathrm{e}} / \mathrm{g}^{\mathrm{g}}$, and ethanol titers at 15.7 and $20.97 \mathrm{~g} / \mathrm{L}$ respectively (Table 5). The remarkable difference between YNB and YP media is the increased accumulation of acetic acid toward YNB from 14 to $53 \mathrm{mg}$ acetic / g glycerol in semi-aerobic conditions (Tables 4, 5). Therefore, the total conversions from the YNB medium reached $90 \%$ of the theoretical value regardless of glucose utilized for cell propagation (Table 5). Discuss the results presented here with the introductory reported study (17) is incomplete due to the differences in genetic backgrounds, experimental aims, and the comparable data. Further studies are needed to clarify whether competing aldehyde dehydrogenase (ALD) with GDH on $\mathrm{NAD}^{+}$is the cause and which ALD isoform is responsible for this accumulation.

The content of amino acids in the defined medium has a crucial role in cell growth (44); furthermore, we observed biosynthesis of $\mathrm{NAD}^{+}$from tryptophan through the kynurenine pathway $(45,46)$. Nicotinic acid, nicotinamide, quinolinic acid, and nicotinamide riboside can salvage the $\mathrm{NAD}^{+}$biosynthesis (28). In this regard, nicotinic acid is auxotrophic under anaerobic conditions in S. cerevisiae (46). We also observed different growth and fermentation with the supplements of amino acids or the buffers using SK-FGG strain (Data not shown). These limitations may explain these lower production titers, conversion rates, and efficiency when 
using YNB compared with the YP medium. We must emphasize that strain SK-FGG was unable to ferment glycerol under the strictly anaerobic condition in our experiment, which used YNB medium (Table 5). Undoubtedly, there are no shuttles for the renovation of NADH under these conditions. Hence, recycling $\mathrm{NADH} / \mathrm{NAD}^{+}$represents the complement element for the robust oxidation of the DHA pathway and efficient utilizing glycerol to produce bioethanol. We are currently working on further engineering for glycerol fermentation under anaerobic conditions while using the high reduction merit of glycerol for improving the fermentation efficiencies of lignocellulose's sugars. Besides, determining the amino acids that may play a significant role with defined media.

In summary, we show here the efficient modeling of glycerol traffic to ethanol production in S. cerevisiae. This systematic metabolic engineering includes integration of the following: (i) imposing robust expression of all genes in the DHA pathway; (ii) prevalence of glycerol oxidation by an oxygen-dependent dynamic by the water-forming NADH oxidase LINoxE, which controls the reaction stoichiometries with the regeneration of the cofactor $\mathrm{NAD}^{+}$; (iii) revoking the first step of both glycerol biosynthesis and glycerol catabolism through G3P. Our study provides innovative addition to metabolic engineering of re-routing glycerol traffic in $S$. cerevisiae while tracking ethanol production to levels that have not yet been attained within any other safe model organisms, either native or genetically engineered $(13,17,18,47-49)$. The metabolic engineering strategy represents another pivotal step for fermenting glycerol in several promising biorefinery scenarios using the glycell process.

\section{ACKNOWLEDGMENTS}

Funding: This work was supported by Mission 5-2 Research Grant from the Research Institute for Sustainable Humanosphere, Kyoto University. 
Author contributions: S.M.R.K. was responsible for the research idea, conception, planning and organisation of the experiments. S.M.R.K. also provided the information for purchasing the strains, chemicals, and toolboxes for the genetic engineering, performed the experiments and analysed and discussed the results. S.M.R.K. wrote, revised, and submitted the manuscript. T.W. was responsible for all financial support and provided all chemicals and equipment. T.W. was involved in the research idea, the conception, the planning and organisation, the discussion of the results and the manuscript revision and submission.

\section{Competing interests:}

The authors state that there are no competing interests to declare.

\section{References}

1- Khattab SMR, Watanabe T. 2019. Bioethanol from sugarcane bagasse: Status and perspectives, p 187-212. In Ramesh CR, Ramachandran S (eds), Bioethanol Production from Food Crops: Sustainable Sources, Interventions, and Challenges, Elsevier. https://doi.org/10.1016/B978-0-12-813766-6.00010-2.

2- Xiberras J, Klein M, Nevoigt E. 2019. Glycerol as a substrate for Saccharomyces cerevisiae based bioprocesses - Knowledge gaps regarding the central carbon catabolism of this 'nonfermentable' $\quad$ carbon $\quad$ source. Biotechnol. $\quad$ Advances, 37(6): 107378. https://doi.org/10.1016/j.biotechadv.2019.03.017.

3- Khattab SMR, Saimura M, Kodaki T. 2013. Boost in bioethanol production using recombinant Saccharomyces cerevisiae with mutated strictly NADPH-dependent xylose reductase and $\mathrm{NADP}\left({ }^{+}\right)$-dependent xylitol dehydrogenase. J biotechnol. 165(3-4): 153-156. https://doi.org/10.1016/j.jbiotec.2013.03.009. 
4- Khattab SMR, Kodaki T. 2014. Efficient bioethanol production by overexpression of endogenous Saccharomyces cerevisiae xylulokinase and NADPH-dependent aldose reductase with mutated strictly $\mathrm{NADP}^{+}$-dependent Pichia stipitis xylitol dehydrogenase. Process Biochem 49: 1838-1842. https://doi.org/10.1016/j.procbio.2014.07.017.

5- Henningsen BM, Hon S, Covalla SF, Sonu C, Argyros DA, Barrett TF, Wiswall E, Froehlich AC, Zelle RM. 2015. Increasing anaerobic acetate consumption and ethanol yields in Saccharomyces cerevisiae with NADPH-specific alcohol dehydrogenase. Appl Environ Microbiol 81(23):8108-17. https://doi.org/10.1128/AEM.01689-15.

6- Nomanbhay S, Hussein R, Ong MY. 2018. Sustainability of biodiesel production in Malaysia by production of bio-oil from crude glycerol using microwave pyrolysis: a review. Green Chem Lett Rev 11: 135-157. https://doi.org/10.1080/17518253.2018.1444795.

7- Yazdani SS, Gonzalez R. 2007. Anaerobic fermentation of glycerol: a path to economic viability for the biofuels industry. Curr Opin Biotechnol 18: 213-219. https://doi.org/10.1016/j.copbio.2007.05.002.

8- Sprague GF, Cronan JE. 1977. Isolation and characterization of Saccharomyces cerevisiae mutants defective in glycerol catabolism. J Bact 129(3): 1335-1342. https://doi.org/10.1128/JB.129.3.1335-1342.1977.

9- Ansell R, Granath K, Hohmann, S, Thevelein JM, Adler L. 1997. The two isoenzymes for yeast $\mathrm{NAD}^{+}$-dependent glycerol 3-phosphate dehydrogenase encoded by GPD1 and GPD2 have distinct roles in osmoadaptation and redox regulation. The EMBO J 16(9): 2179-2187. https://doi.org/10.1093/emboj/16.9.2179.

10-Grauslund M, Lopes JM, Rønnow B. 1999. Expression of GUT1, which encodes glycerol kinase in Saccharomyces cerevisiae, is controlled by the positive regulators Adr1p, Ino2p and 
Ino4p and the negative regulator Opi1p in a carbon source-dependent fashion. Nucleic acids Res 27(22): 4391-4398. https://doi.org/10.1093/nar/27.22.4391.

11-Grauslund M, Rønnow B. 2000. Carbon source-dependent transcriptional regulation of the mitochondrial glycerol-3-phosphate dehydrogenase gene, GUT2, from Saccharomyces cerevisiae. Can J Microbiol 46(12): 1096-1100. https://doi.org/10.1139/w00-105

12-Turcotte B, Liang XB, Robert F, Soontorngun N. 2010. Transcriptional regulation of nonfermentable carbon utilization in budding yeast. FEMS yeast res 10(1): 2-13. https://doi.org/10.1111/j.1567-1364.2009.00555.x.

13- Yu KO, Kim SW, Han SO. 2010. Engineering of glycerol utilization pathway for ethanol production by Saccharomyces cerevisiae. Bioresour Technol 101(11): 4157-4161. https://doi.org/10.1016/j.biortech.2010.01.066.

14-Swinnen S, Klein M, Carrillo M, McInnes J, Nguyen H, Nevoigt E. 2013. Re-evaluation of glycerol utilization in Saccharomyces cerevisiae: characterization of an isolate that grows on glycerol without supporting supplements. Biotechnol Biofuels 6(1): 157. https://doi.org/10.1186/1754-6834-6-157.

15-Swinnen S, Ho PW, Klein M, Nevoigt E. 2016. Genetic determinants for enhanced glycerol growth of Saccharomyces cerevisiae. Met $\quad$ Eng 36: $\quad$ 68-79. https://doi.org/10.1016/j.ymben.2016.03.003.

16- Klein M, Carrillo M, Xiberras J, Islam ZU, Swinnen S, Nevoigt E. 2016. Towards the exploitation of glycerol's high reducing power in Saccharomyces cerevisiae-based bioprocesses. Met Eng 38: 464-472. https://doi.org/10.1016/j.ymben.2016.10.008.

17-Aßkamp MR, Klein M, Nevoigt E. 2019. Saccharomyces cerevisiae exhibiting a modified route for uptake and catabolism of glycerol forms significant amounts of ethanol from this 
carbon source considered as 'non-fermentable'. Biotechnol Biofuels 12:257. https://doi.org/10.1186/s13068-019-1597-2.

500 18-Semkiv M, Kata I, Ternavska O, Sibirny W, Dmytruk K, Sibirny A. 2019. Overexpression of 501 the genes of glycerol catabolism and glycerol facilitator improves glycerol conversion to 502 ethanol in the methylotrophic thermotolerant yeast Ogataea polymorpha. Yeast 36(5): 329339. https://doi.org/10.1002/yea.3387.

504 19-Liu J, Takada R, Karita S, Watanabe T. Honda Y, Watanabe T. 2010. Microwave-assisted 505 pretreatment of recalcitrant softwood in aqueous glycerol. Bioresour technol 101(23): 93559360. https://doi.org/10.1016/j.biortech.2010.07.023.

20- Ohashi Y, Watanabe T. 2018. Catalytic Performance of Food Additives Alum, Flocculating Agent, $\mathrm{Al}\left(\mathrm{SO}_{4}\right)_{3}, \mathrm{AlCl}_{3}$, and Other Lewis Acids in Microwave Solvolysis of Hardwoods and Recalcitrant Softwood for Biorefinery. ACS omega 3(11): 16271-16280.

511 21-Kang YS, Kane J, Kurjan J, Stadel JM, Tipper DJ. 1990. Effects of expression of mammalian $\mathrm{G}$ alpha and hybrid mammalian-yeast $\mathrm{G}$ alpha proteins on the yeast pheromone response

$514 \quad$ https://doi.org/10.1128/mcb.10.6.2582.

515 22-Ito Y, Kitagawa T, Yamanishi, Katahira S, Izawa S, Irie K, Furutani-Seiki M, Matsuyama T. 516 2016. Enhancement of protein production via the strong DIT1 terminator and two RNA- 
519

520

521

522

523

524

525

526

527

528

529

530

531

532

23-Ryan OW, Skerker JM, Maurer MJ, Li X, Tsai JC, Poddar S, Lee ME, DeLoache W, Dueber JE, Arkin AP, Cate JH. 2014. Selection of chromosomal DNA libraries using a multiplex CRISPR system. eLife 3: e03703. https://doi.org/10.7554/eLife.03703.

24-Naito Y, Hino K, Bono H, Ui-Tei K. 2015. CRISPRdirect: software for designing CRISPR/Cas guide RNA with reduced off-target sites. Bioinformatics 31(7): 1120-1123. https://doi.org/10.1093/bioinformatics/btu743.

25-Hosaka K, Nikawa J, Kodaki T, Yamashita S. 1992. A dominant mutation that alters the regulation of INO1 expression in Saccharomyces cerevisiae. J Biochem 111(3): 352-358. https://doi.org/10.1093/oxfordjournals.jbchem.a123761.

26- Ryan OW, Poddar S, Cate JH. 2016. CRISPR-Cas9 genome engineering in Saccharomyces cerevisiae cells. Cold Spring Harbor protocols, 2016(6), 10.1101/pdb.prot086827. https://doi.org/10.1101/pdb.prot086827.

27- Nguyen H T, Nevoigt E. 2009. Engineering of Saccharomyces cerevisiae for the production of dihydroxyacetone (DHA) from sugars: a proof of concept. Met Eng 11(6): 335-346. https://doi.org/10.1016/j.ymben.2009.07.005.

28-Plaut B, Knowles JR. 1972. pH-dependence of the triose phosphate isomerase reaction. Biochem J 129(2): 311-320. https://doi.org/10.1042/bj1290311.

29-Hosaka K, Murakami T, Kodaki T, Nikawa J, Yamashita S. 1990. Repression of choline kinase by inositol and choline in Saccharomyces cerevisiae. J Bacteriol Res 172(4): 20052012. https://doi.org/10.1128/jb.172.4.2005-2012.1990.

30-Kodaki T, Yamashita S. 1989. Characterization of the methyltransferases in the yeast phosphatidylethanolamine methylation pathway by selective gene disruption. Eur J Biochem 185(2): 243-251. https://doi.org/10.1111/j.1432-1033.1989.tb15109.x. 
31-Vemuri GN, Eiteman MA, McEwen JE, Olsson L, Nielsen J. 2007. Increasing NADH oxidation reduces overflow metabolism in Saccharomyces cerevisiae. Proc Natl Acad Sci USA 104(7): 2402-2407. https://doi.org/10.1073/pnas.0607469104.

32- Kim J-W, Seo S-O, Zhang G-C, Jin Y-S, Seo J-H. 2015. Expression of Lactococcus lactis $\mathrm{NADH}$ oxidase increases 2,3-butanediol production in Pdc-deficient Saccharomyces cerevisiae. Bioresour Technol 191: 512-519. https://doi.org/10.1016/j.biortech.2015.02.077.

33- Kim S, Hahn JS, 2015. Efficient production of 2,3-butanediol in Saccharomyces cerevisiae by eliminating ethanol and glycerol production and redox rebalancing. Met Eng 31: 94-101. https://doi.org/10.1016/j.ymben.2015.07.006.

34-Bae SJ, Kim S, Hahn JS. 2016. Efficient production of acetoin in Saccharomyces cerevisiae by disruption of 2,3-butanediol dehydrogenase and expression of NADH oxidase. Sci Rep. 6: 27667. https://doi.org/10.1038/srep27667.

35-Kim JW, Lee YG, Kim SJ, Jin YS, Seo JH. 2019. Deletion of glycerol-3-phosphate dehydrogenase genes improved 2,3-butanediol production by reducing glycerol production in pyruvate decarboxylase-deficient Saccharomyces cerevisiae. J biotechnol 304: 31-37. https://doi.org/10.1016/j.jbiotec.2019.08.009.

36- Murata K, Fukuda Y, Watanabe K, Saikusa T, Shimosaka M, Kimura A. 1985. Characterization of methylglyoxal synthase in Saccharomyces cerevisiae. Biochem Biophys Res Commun 131(1): 190-198. https://doi.org/10.1016/0006-291x(85)91788-7.

37-Zheng Z, Zou J. 2001. The initial step of the glycerolipid pathway: identification of glycerol 3-phosphate/dihydroxyacetone phosphate dual substrate acyltransferases in Saccharomyces cerevisiae. J biol Chem 276(45): 41710-41716. https://doi.org/10.1074/jbc.M104749200. 
38-Overkamp KM, Bakker BM, Kötter P, Luttik MA, Van Dijken JP, Pronk JT. (2002). Metabolic engineering of glycerol production in Saccharomyces cerevisiae. Appl Environ Microbiol 68(6): 2814-2821. https://doi.org/10.1128/aem.68.6.2814-2821.2002.

39- Yamada-Onodera K, Yamamoto H, Emoto E, Kawahara N, Tani Y. 2002. Charaterisation of glycerol dehydrogenase from a methylotrophic yeast Hansenula polymorpha DL-1, and its gene cloning. Acta. Biotechnol. 22: 337-353. https://doi.org/10.1002/15213846(200207)22:3/4<337::AID-ABIO337>3.0.CO;2-6.

40-Molin M, Norbeck J, Blomberg A. 2003. Dihydroxyacetone kinases in Saccharomyces cerevisiae are involved in detoxification of dihydroxyacetone. J biol chem 278(3): 14151423. https://doi.org/10.1074/jbc.M203030200.

41-Lutstorf U, Megnet R. 1968. Multiple forms of alcohol dehydrogenase in Saccharomyces cerevisiae. I. Physiological control of ADH-2 and properties of ADH-2 and ADH-4. Arch Biochem Biophys 126(3): 933-944. https://doi.org/10.1016/0003-9861(68)90487-6.

42-Ito Y, Yamanishi M, Ikeuchi A, Imamura C, Tokuhiro K, Kitagawa T, Matsuyama T. 2013. Characterization of five terminator regions that increase the protein yield of a transgene in Saccharomyces cerevisiae. $\mathrm{J}$ biotechnol 168(4): 486-492. https://doi.org/10.1016/j.jbiotec.2013.09.024.

43-Wei L, Wang Z, Zhang G, Ye B. 2017. Characterization of terminators in Saccharomyces cerevisiae and an exploration of factors affecting their strength. Chem Bio Chem 18(24): 2422-2427. https://doi.org/10.1002/cbic.201700516.

44-Roberts TM, Kaltenbach H-M, Rudolf F. 2020. Development and optimisation of a defined high cell density yeast medium. Yeast 37(5-6): 336-347. https://doi.org/10.1002/yea.3464. 
45-Panozzo C, Nawara M, Suski C, Kucharczyka R, Skoneczny M, Bécam AM, Rytka J, Herbert CJ. 2002. Aerobic and anaerobic $\mathrm{NAD}^{+}$metabolism in Saccharomyces cerevisiae. FEBS lett 517(1-3): 97-102. https://doi.org/10.1016/s0014-5793(02)02585-1.

46- Kato M, Lin SJ. 2014. Regulation of $\mathrm{NAD}^{+}$metabolism, signaling and compartmentalization in the yeast Saccharomyces cerevisiae. DNA Repair 23: 49-58.

$$
\text { https://doi.org/10.1016/j.dnarep.2014.07.009. }
$$

47- Yazdani SS, Gonzalez R. 2008. Engineering Escherichia coli for the efficient conversion of glycerol to ethanol and co-products. Met. Eng. 10(6): 340-351. https://doi.org/10.1016/j.ymben.2008.08.005.

48-Trinh CT, Srienc F. 2009. Metabolic engineering of Escherichia coli for efficient conversion of glycerol to ethanol. Appl Environ Microbiol 75: 6696-6705. https://doi.org/10.3389/fbioe.2014.00016.

49-Loaces I, Rodríguez C, Amarelle V, Fabiano E, Noya F. 2016. Improved glycerol to ethanol conversion by $E$. coli using a metagenomic fragment isolated from an anaerobic reactor. J Ind Microbiol Biotecnol 43(10): 1405-1416. https://doi.org/10.1007/s10295-016-1818-7.

\section{Legends of figures and tables}

Fig. 1. Schematic diagram showing the integrative scenario of a biorefinery with new generation of glycerol fermenting yeast and redirection of glycerol influxes to ethanol production in Saccharomyces cerevisiae via retrofitted native glycerol anabolic and catabolic pathways using the robust oxidative route with renovation of the $\mathrm{NAD}^{+}$cofactor via $\mathrm{O}_{2}$ dependent dynamics of water-forming NADH oxidase. During pathway re-routing, glycerol3-phosphate dehydrogenase $1(S c G P D 1)$ and glycerol kinase 1 (GUT1) were knocked-out. A highlighted circle indicates the overexpressed indigenous $S$. cerevisiae enzymes 
609 dihydroxyacetone kinase $(S c D A K) 1$ and 2 as well as triosephosphate isomerase $(S c T P I) 1$,

610 heterologous glycerol dehydrogenase from Ogataea polymorpha (OpGDH), glycerol

611 facilitator from Candida utilis (CuFPS1) and water-forming NADH oxidase from

612 Lactococcus lactis subsp. lactis Il1403 (LINoxE).

613

614 Fig. 2. Depicted module M1 for replacing $S c G U T 1$ by homologous recombination using

615 Multiplex CRISPR Cas 9. 
617 Fig. 3. Comparison of the time course of glycerol-glucose fermentation between the $S$. cerevisiae strains within this study:

618 Reference ancestral strain (black lines); NOXE strain (orange lines); GDH strain (green lines); GN strain (purple lines); GN-FDT

619 strain (blue lines); GN-FDT-M1[SK-FGG] (red lines); (a) Glucose consumption (rhomboid symbols), glycerol consumption

620 (squares); (b) ethanol production (triangles). Fermentation carried out in semi-aerobic conditions in flasks with 20:200 medium:

621 flask volume at $30^{\circ} \mathrm{C}$ with shaking at $180 \mathrm{rpm}$. YP medium supplemented with (w/v) $1.5 \%$ glucose, and $7 \%$ glycerol was used. (n $622=3)$

623 
Table 1. Characteristics of $S$. cerevisiae strains generated in this study:

S. cerevisiae strain

D452-2

D452-2-URA3

GDH

GPD1/LINoxE (NOXE)

GDH-NOXE (GN)

D452-2-URA3-AUR1-C

GN-FDT

GN-FDT-M1 (SK-FGG)

\section{Relevant genotype}

MATa leu2 his3 ura3 can1

D452-2, URA3:: TDH3 promoter and d22DIT1 terminator

D452-2, URA3:: TDH3p-OpGDH-d22-DIT1t

D452-2, $\Delta$ GPD1:: TDH3p-LlNoxE-d22-DIT1t

D452-2, URA3:: TDH3p-OpGDH-d22-DIT1t; $\triangle G P D 1::$ TDH3p-LINoxE-d22-DIT1t

D452-2, URA3:: TDH3 promoter and d22DIT1 terminator :: AUR1-C

D452-2, URA3:: TDH3p-OpGDH-d22-DIT1t; $\triangle G P D 1::$ TDH3p-LINoxE-d22-DIT1t; AUR1-

C:: PGKp-CuFPS1-RPL41Bt; PGKp-ScTPI1-PGKt; PGKp-ScDAK2-PGKt; PGKp-ScDAK1- This study PGKt

D452-2, URA3:: TDH3p-OpGDH-d22-DIT1t; $\triangle G P D 1::$ TDH3p-LINoxE-d22-DIT1t; AUR1C:: PGKp-CuFPS1-RPL41Bt; PGKp-ScTPI1-PGKt; PGKp-ScDAK2-PGKt; PGKp-ScDAK1PGKt; $\triangle G U T 1:: \quad$ TEFp-CuFPS1-CYC1t; TYS1p-OpGDH-ATP15t; TDH3p-ScDAK1-d22-

DIT1t; FBA1p-ScTPI1-TDH3t

\section{Reference}

This study

This study

this study

This study

This study

This study 
Table 2: Constructed plasmids in this study.

\begin{tabular}{|c|c|c|}
\hline Plasmid & Relevant genotype & Reference \\
\hline pPGK-URA3 & URA3; PGK promoter and terminator & $(21)$ \\
\hline pPGK-DAK1 & URA3; expresses DAK1 & This study \\
\hline pPGK-DAK2 & URA3; expresses DAK2 & This study \\
\hline pPGK-TPI1 & URA3; expresses TPI1 & This study \\
\hline pPGK-TPI1, ScDAK2 & URA3; expresses TPI1, DAK2 & This study \\
\hline pPGK-TPI1, ScDAK2, ScDAK1 & URA3; expresses TPI1, DAK2, DAK1 & This study \\
\hline TDH3p-d22DIT1t- URA3 & URA3, TDH3 promoter and d22DIT1 terminator & This study \\
\hline TDH3-OpGDH-d22DIT1t-URA3 & URA3; expresses $O p \mathrm{GDH}$ & This study \\
\hline TDH3-L1NoxE-d22 DIT1t-URA3 & URA3; expresses $L l$ NoxE & This study \\
\hline pAUR101 & AUR1-c & Takara Bio \\
\hline pAUR101-PGKp-RPL41Bt & AUR1-C, PGKp-RPL41Bt & This study \\
\hline pAUR101-PGKp-CuFPS1-RPL41Bt & AUR1-C; expresses $C u$ FPS1 & This study \\
\hline pAUR101-CuFPS1, TPI1, DAK2, DAK1 & AUR1-C; expresses $C u$ FPS1, TPI1, DAK2, DAK1 & This study \\
\hline pAUR101- M1* & AUR1-C; expresses $C u$ FPS1, $O p$ GDH, DAK1, TPI1 & This study \\
\hline
\end{tabular}


Multiplex pCAS (Addgene \#60847)

Multiplex pCAS/GPD1-1

Multiplex pCAS/GPD1-2

Multiplex pCAS/GUT1
Multiplex expresses Cas9 and HDV ribozyme-sgRNA

Multiplex expresses Cas9 and gRNA to base No. 135 of GPD1 This study

Multiplex expresses Cas9 and gRNA to base No. 1045 of GPD1 This study

Multiplex expresses Cas9 and gRNA to base No. 616 of GUT1

This study

*M1: TEFp-CuFPS1-CYC1t; TYS1p-OpGDH-ATP15t; TDH3p-ScDAK1-d22-DIT1t; FBA1p-ScTPI1-TDH3t

629 
Table 3. Specific activities of the enzymes; glycerol dehydrogenase $(G D H)$, dihydroxyacetone kinase $(D A K)$, triosephosphate isomerase $(T P I I)$ and $\mathrm{NADH} / \mathrm{NAD}^{+}$ratio with their intracellular concentrations in the recombinant strains in this study. Error values represent standard deviation of the mean $(n=2)$.

* indicates not detected. $\quad \propto$ indicates not measured.

634

\begin{tabular}{|c|c|c|c|c|c|c|}
\hline Relevant & Enzyme ac & $\begin{array}{l}\text { vities } \mu \text { mole } \\
\text { tracted prote }\end{array}$ & $\mathrm{min} / \mathrm{mg}$ cell & \multicolumn{2}{|c|}{ Intracellular concentrations } & \multirow{2}{*}{$\begin{array}{c}\text { NADH/ NAD } \\
\text { Ratio }\end{array}$} \\
\hline Strain & $G D H$ & $D A K s$ & TPII & $\mathbf{N A D H}(\boldsymbol{\mu M})$ & $\mathbf{N A D}^{+}(\boldsymbol{\mu M})$ & \\
\hline WT & $\mathrm{ND}^{*}$ & $3.9 \pm 0.23$ & $0.058 \pm 0.003$ & $0.17 \pm 0.004$ & $0.36 \pm 0.01$ & $0.47 \pm 0.01$ \\
\hline $\mathrm{GDH}$ & $0.063 \pm 0.001$ & NMă & $0.055 \pm 0.003$ & $0.26 \pm 0.008$ & $0.38 \pm 0.02$ & $0.68 \pm 0.02$ \\
\hline NOXE & ND* & NMă & $0.054 \pm 0.002$ & $0.04 \pm 0.005$ & $0.36 \pm 0.04$ & $0.12 \pm 0.01$ \\
\hline GN & $0.065 \pm 0.004$ & NMa & $0.057 \pm 0.003$ & $0.34 \pm 0.018$ & $0.35 \pm 0.01$ & $0.99 \pm 0.02$ \\
\hline GN-FDT & $0.071 \pm 0.003$ & $7.15 \pm 0.23$ & $0.067 \pm 0.004$ & $0.17 \pm 0.08$ & $0.52 \pm 0.01$ & $0.33 \pm 0.02$ \\
\hline SK-FGG & $0.111 \pm 0.002$ & $18.3 \pm 0.28$ & $0.076 \pm 0.004$ & $0.11 \pm 0.01$ & $0.63 \pm 0.01$ & $0.18 \pm 0.01$ \\
\hline
\end{tabular}



adding glucose.

$637 \quad \#$; Fermentation conditions and products values

\begin{tabular}{|c|c|c|c|c|}
\hline Parameter & $\begin{array}{l}\text { One fed-batch } \\
{[\mathrm{A}]}\end{array}$ & $\begin{array}{l}\text { One fed-batch } \\
\text { [B] }\end{array}$ & $\begin{array}{l}\text { Two fed-batch } \\
\text { [C] }\end{array}$ & $\begin{array}{l}\text { Two fed-batch } \\
\text { [D] }\end{array}$ \\
\hline Fermentation time (h) & $40 \pm 0.30$ & $40 \pm 0.30$ & $96 \pm 0.30$ & $96 \pm 0.30$ \\
\hline Total consumed glycerol (g/L) & $82.5 \pm 1.63$ & $75.77 \pm 1.34$ & $141.88 \pm 2.66$ & $141.703 \pm 4.79$ \\
\hline Total consumed glucose (g/L) & NA & $22.55 \pm 1.14$ & $22.2 \pm 0.67$ & $44.67 \pm 0.9$ \\
\hline Rate of consumption $\left(\mathrm{g} / \mathrm{L}^{-1} \mathrm{~h}^{-1}\right)$ & $2.06 \pm 0.017$ & $2.42 \pm 0.014$ & $1.71 \pm 0.011$ & $1.94 \pm 0.015$ \\
\hline Ethanol yield (g/L) & $40.42 \pm 0.89$ & $48.22 \pm 1.16$ & $79.74 \pm 1.03$ & $86.54 \pm 2.48$ \\
\hline Rate of ethanol production $\left(\mathrm{g} / \mathrm{L}^{-1} \mathrm{~h}^{-1}\right)$ & $1.01 \pm 0.01$ & $1.2 \pm 0.02$ & $0.83 \pm 0.01$ & $0.9 \pm 0.02$ \\
\hline Efficiency of ethanol production $\left(\mathrm{g}^{\mathrm{e}} / \mathrm{g}^{\mathrm{s}}\right) *$ & $0.49 \pm 0.002$ & $0.49 \pm 0.002$ & $0.486 \pm 0.003$ & $0.464 \pm 0.003$ \\
\hline Efficient/ theoretical $(\%)^{+}$ & 98 & 98 & 97.2 & 92.8 \\
\hline Acetic acid accumulation (g/L) & $1.14 \pm 0.02$ & $1.12 \pm 0.02$ & $1.67 \pm 0.1$ & $2.46+0.02$ \\
\hline Total conversion $\left(\mathrm{g}^{\mathrm{p}} / \mathrm{g}^{\mathrm{s}}\right)^{++}$ & $0.5 \pm 0.002$ & $0.5 \pm 0.003$ & $0.496 \pm 0.005$ & $0.478 \pm 0.004$ \\
\hline Cell Density $\left(\mathrm{OD}_{600}\right)$ & $10.6 \pm 0.2$ & $11.6 \pm 0.2$ & $11.6 \pm 0.3$ & $15.2 \pm 0.3$ \\
\hline
\end{tabular}

Oxygen availability in (liquid medium: flask volume) at $30^{\circ} \mathrm{C}$ with shaking at $200 \mathrm{rpm}$ (20:200).

$639 \quad\left(\mathrm{~g}^{\mathrm{e}} / \mathrm{g}^{\mathrm{s}}\right) *$; gram ethanol per gram of glycerol. $\quad\left(\mathrm{g}^{\mathrm{p}} / \mathrm{g}^{\mathrm{s}}\right){ }^{++}$; gram products per gram substrate 
Table 5. Fermentation characteristics of glycerol as a sole carbon source by the generated strain SKFGG at different oxygen availabilities.

\begin{tabular}{lllll}
\hline Parameter name & \multicolumn{4}{c}{ Fermentation conditions and products values } \\
\hline Oxygen availability\# & Strict anaerobic & Micro-aerobic & Semi-aerobic & Aerobic \\
Fermentation time $(\mathrm{h})$ & $96 \pm 0.30$ & $60 \pm 0.30$ & $48 \pm 0.30$ & $36 \pm 0.30$ \\
Total consumed glycerol $(\mathrm{g} / \mathrm{L})$ & $0.00 \pm 0.45$ & $37.17 \pm 2.66$ & $54.01 \pm 4.88$ & $46.45 \pm 0.74$ \\
Rate of consumption $\left(\mathrm{g} / \mathrm{L}^{-1} \mathrm{~h}^{-1}\right)$ & $0.0 \pm 0.0$ & $0.62 \pm 0.4$ & $1.12 \pm 0.037$ & $1.29 \pm 0.035$ \\
Total ethanol produced $(\mathrm{g} / \mathrm{L})$ & $0.0 \pm 0.0$ & $15.71 \pm 1.87$ & $20.97 \pm 1.25$ & $17.97 \pm 0.53$ \\
Rate of ethanol production $\left(\mathrm{g} / \mathrm{L}^{-1} \mathrm{~h}^{-1}\right)$ & $0.0 \pm 0.0$ & $0.25 \pm 0.04$ & $0.44 \pm 0.02$ & $0.5 \pm 0.01$ \\
Efficiency of ethanol production $\left(\mathrm{g}^{\mathrm{e}} / \mathrm{g}^{\mathrm{s}}\right)$ & $0.0 \pm 0.0$ & $0.42 \pm 0.05$ & $0.39 \pm 0.01$ & $0.39 \pm 0.01$ \\
Efficient/ theoretical $(\%)$ & 0 & 84 & 78 & 78 \\
Acetic acid accumulation $(\mathrm{g} / \mathrm{L})$ & $0.0 \pm 0.0$ & $0.87 \pm 0.21$ & $2.88 \pm 0.19$ & $3.04 \pm 0.09$ \\
Total conversions $\left(\mathrm{g}^{\mathrm{p}} / \mathrm{g}\right)$ & $0.0 \pm 0.0$ & $0.45 \pm 0.04$ & $0.44 \pm 0.01$ & $0.45 \pm 0.01$ \\
Cell density $\left(\mathrm{OD}_{600}\right)$ & $4.66 \pm 0.1$ & $8.55 \pm 0.27$ & $8.55 \pm 0.1$ & $9 \pm 0.1$ \\
\hline
\end{tabular}

